\title{
ALGUNOS BRIOFITOS INTERESANTES PARA LA FLORA BALEAR
}

\author{
R.M. CROS (*)
}

\section{RESUMEN}

Se dan a conocer algunas especies interesantes para las Baleares y también alguna especie nueva para la Flora insular como Riella belicophylla Mont.

\section{SUMMARY}

Some interesting species for the Balearic Islands are brought to light, as well as one or two species new to the Iberian insular flora such as Riella belicophylla Mont.

En el estudio briológico de las islas Baleares, han contribuido Cambessedes, Barcelo, Rodríguez Femenias, Nicholson, Knoche, Maheu, Dixon, Casas, Koppe, Sloover, Vives y Dunk principalmente, pero estos autores centraron su estudio en las islas de Mallorca y Menorca; actualmente se tiene un conocimiento de estas islas bastante completo, seguramente por ser éstas más ricas en briófitos que las Pitiusas. Sobre estas i slas sólo encontramos en la bibliografía un estudio de los briófitos de las cuevas de Ibiza hecho por Maheu (1912) en el que figuran 5 especies de musgos. Posteriormente Casas (1966) hace un estudio de los briófitos recogidos en las Pitiusas por la Sra.de Bolos, en este trabajo se citan 27 briófitos para Ibiza y 5 para Formentera; Jovet-Ast y Bischler (1976) enumeran 7 hepáticas para Formentera y 1 para Ibiza.

Desde el punto de vista pedológico, las Pitiusas están formadas enteramente por sedimentos calcáreos. Las s uperficies calizas captan el agua de lluvia, reteniéndola en 1 as profundidades del carst, o sea desaparece rápidamente el agua y la humedad, lo que uniformiza las condiciones a mbientales prévalenciendo las condiciones xerofíticas.

Las temperaturas no sufren variaciones importantes, oscilan entre $l$ os $10^{\circ} \mathrm{y}$ los $25^{\circ} \mathrm{C}$ siendo la media anual de unos $17^{\circ} \mathrm{C}$.

A diferencia de las islas de Mallorca y Menorca que sufren el efecto de la "tramuntana", las Pitiusas están sometidas a un régimen débil de vientos.

El relieve es suave y el paisaje está formado por pequeñas colinas, Ibiza alcanza los $415 \mathrm{~m}$. de altitud y Formentera solamente $192 \mathrm{~m}$, por lo que el gradiente de variación de la flora es débil.

Comunicación presentada al III Simposio Nacional de Botánica Criptogámica. Málaga, 1978

(1) Departamento de Botánica, Fac. de Ciencias. Universidad Autónoma de Barcelona. 
El clima es eminentemente mediterráneo septentrional, cosa que se refleja en la vegetación superior y también en los briófitos.

En el mes de Mayo de este año tuve ocasión de visitar Ibiza durante tres días y uno en Formentera. Se recolectaron 125 muestras en Ibiza y 31 en Formentera; éstas se hicieron en Ibiza en acantilados rocosos a provechando las grietas más húmedas, en torrentes actualmente secos pero umbríos, en claros de bosque y en el río Sta. Eulalia como único curso de agua de la isla. En Formentera, de clima más seco todavía, las recolecciones se hicieron en grietas de acantilados y en los claros del bosque.

El número de especies determinadas, así como la presencia de alguna de ellas en $l$ as Pitiusas, hace que aumente su área de distribución, hechos éstos que motivaron la publicación de esta nota. Una especie interesante es la hepática Riella belicophylla Mont., Sphaerocarpaceae, hidrófila, halófila que en España habita en los bordes y en el fondo de las lagunas saladas en las zonas endorreicas, aquí la encontramos en el río Sta. Eulalia a unos $100 \mathrm{~m}$ de la desembocadura y semisumergida en el agua. En los ejemplares recolectados se observan claramente anteridios, arquegonios y esporogonios con esporas maduras de $81-95 \mathrm{~nm}$ de diámetro y ornamentadas con espinas de 9,13 nm de longitud con sus extremos algo truncados.

La distribución de esta especie es mediterráneo-atlántica; se encontró por primera vez en Argelia cerca de Orán por Montagne en 1844, posteriormente se ha citado en Túnez en los mares de la Sénia (Allorge 1929); en España cerca de Toledo (Allorge 1936) y en Los Monegros (Casas 1970); en Palestina cerca de Jerusalén (Washburn \& Jones 1937); en Portugal en la región de Algarve (Tavares 1952); en Egipto cerca de El Cairo (Persson \& Imam 1960) y en Francia al SW de la cuenca del Thau (Dubois et Hebant 1968). (Ver mapa de distribución).

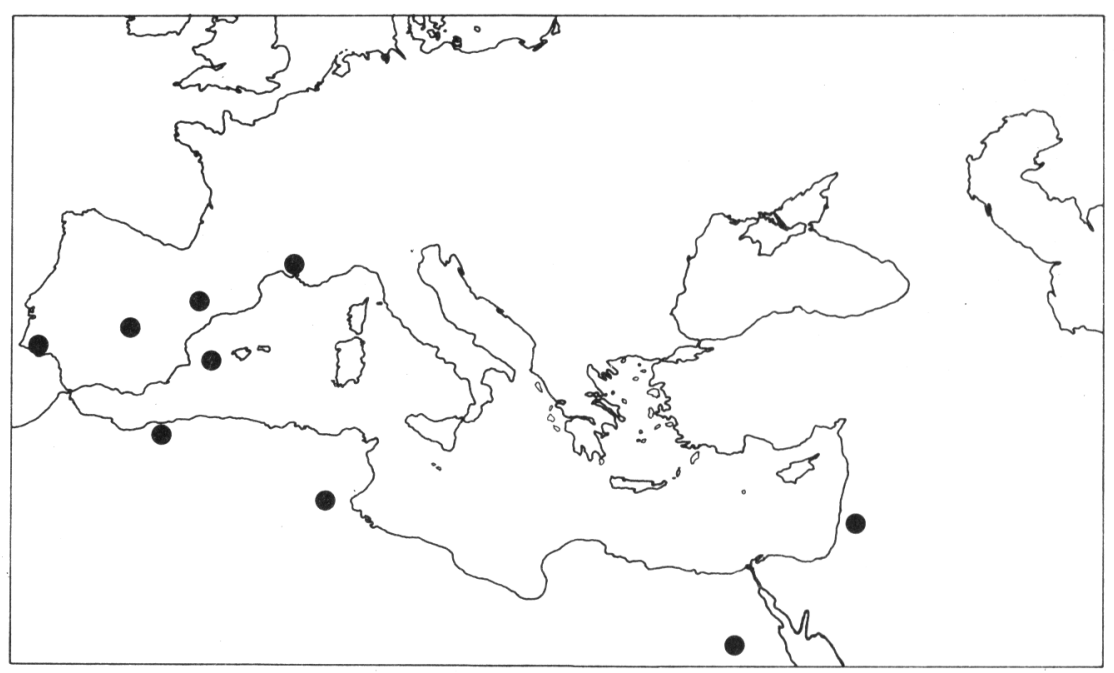

Distribución de Riella belicophylla Mont. en los países mediterráneos, según autores indicados. 
Además de esta hepática, en Ibiza se encontró Gyroweisia tenuis (Hedw.) Schimp. que tampoco había sido citada en las Baleares; se citan también 5 hepáticas y 19 musgos nuevos para las Pitiusas.

En Formentera, como novedad para las Baleares, podemos citar Fissidens exilis Hedw. que a pareció con la hepática Fossombronia sp. que por encontrarse en estado estéril no se pudo determinar la especie; figuran además en el catálogo 14 musgos no citados en Formentera de los cuales 8 son nuevos para las Pitiusas.

Las especies precedidas por " son citas nuevas para las Pitiusas y las precedidas por $* *$ son citas nuevas para las Baleares.

Resumiendo, el total de briófitos encontrados hasta ahora son: 12 hepáticas y 43 musgos en Ibiza y 9 hepáticas y 18 musgos en Formentera. En el estudio de las muestras estudiadas observamos poca diversidad de especies, la caus a debe radicar en la uniformidad de hábitats unido a la aridez del clima por lo que encontramos muy pocos epífitos.

Knoche (1921) en su Flora Balearica dice que en Ibiza los musgos son muy raros por lo que ni los hace constaren sus notas. A pesar de esto creo que en posteriores recolecciones se puede no sólo aumentar el catálogo de especies sino también encontrar especies interesantes para la Flora briológica balear.

El catálogo de especies incluye para Ibiza 12 hepáticas y 38 musgos, y para Formentera 2 hepáticas y 16 musgos. La ordenación de los musgos se ha hecho según. Smith (1978) y las hepáticas según Müller (1954).

\section{IBIZA}

\section{HEPATICAS}

Riella helicophylla Mont.

Río Sta. Eulalia. Talud del río semisumergida. Ejemplares con a nteridios, arquegonios y esporogonios. Esporas de 81-95 nm. Espinas de 9,13 nm.

Lunaria cruciata (L.) Dum.

Cala d'Aubarca. Claros en el Rosmarino-Ericion.

Fossombronia caespitiformis De Not.

Cala Pou des Lleó, rambla con Juniperus phoenicea v. lycia y Nerium oleander.

\section{Fossombronia sp.}

Los ejemplares encontrados no estaban fructificados por lo que no se ha podido determinar la especie. Cala d'Aubarca sobre rocas. Torrent de l'Escalonada sobre rocas umbrías y sobre suelo.

Leiocolea turbinata (Raddi) Buch.

Torrent de l'Escalonada sobre rocas y en las fisuras. Cala d'Aubarca talud muy húmedo al pie del acantilado y en un talud. Cala Entrepenyes acantilado que rezuma agua. 
Southbya tophacea Spruce.

Cala d'Aubarca bajo Rosmarinus officianalis y Erica multiflora talud muy húmedo al pie del acantilado. Torrent de l'Escalonada sobre suelo, sobre rocas y en las fisuras.

* Southbya nigrella (De Not.) Spr.

Cala Pou des Lleó, rambla con J. phoenicea v. bycia y $N$. oleander.

Cephaloziella baumgartneri Schiffn.

Puig d'en Corda sobre suelo en el Rosmarino-Ericion con pinos. Cala Pou des Lleó sobre suelo en la rambla con J. phoenicea v. Lycia y $N$. oleander.

Cephaloziella starkei (Funk) Schiffn.

Cala d'Aubarca bajo R. officinalis y E. multiflora en un talud con Soutbbya tophacea y Gymnostomum calcareum.

* Frullania dilatata Dum.

Torrent de l'Escalonada sobre piedras.

Lejeunea cavifolia (Ehrh.) Ldbg.

Torrent de l'Escalonada sobre rocas.

Lejeunea patens Ldbg.

Torrent de l'Escalonada sobre rocas y epífito sobre $P$. halepensis y J.phoeniceav. bycia.

\section{MUSGOS}

* Dicranella varia (Hedw.) Schimp. var. tenuifolia (=D. howei).

Cala d'Aubarca debajo de $R$. officinalis y E. multiflora y sobre rocas al pie del acantilado en un talud húmedo. Río Sta. Eulalia, sobre suelo.

* Fissidens incurvus Starke ex Röhl.

Puig Tanques sobre pared artificial.

Tortula muralis Hedw.

Torrent de l'Escalonada sobre piedras. Cala d'Aubarca sobre rocas y al pie de rocas húmedas.

Aloina rigida (Hedw.) Limpr.

Cala Pou des Lleó, sobre suelo en la rambla con J. phoenicea v. lycia y N. oleander.

* Aloina aloides (Schultz.) Kindb. var. ambigua (Br. Eur.) Craig in Grout. Puig Tanques sobre pared.

Pottia sp.

Ejemplar sin fructificar en Torrent de l'Escalonada sobre piedras. 
* Barbula ungiculata Hedw.

Puig Tanques sobre pared. Cala Pou des Lleó sobre suelo en la rambla con $I$. phoenicea v. lycia y $N$. oleander.

Barbula fallax Hedw.

Cala d'Aubarca debajo de R. officinalis y E. multiflora.

Barbula tophacea (Brid.) Mitt.

Río Sta. Eulalia sobre suelo. Cala d'Aubarca al pie de rocas.

Barbula vinealis Brid.

Cala d'Aubarca en talud húmedo al pie del acantilado, bajo $R$. officinalis y $E$. multiflora y en los claros del Rosmarino-Ericion sobre el suelo. Torrent de l'Escalonada sobre rocas. Cala d'en Sardina. Puig d'en Corda. Cala Pou des Lleó en la rambla con J. phoenicea v. lycia y $N$. oleander.

Gymnostomum calcareum Nees \& Hornsch.

Cala d'Aubarca sobre rocas y sobre suelo debajo de $R$. officinal is y E. multiflora; en un talud. Torrent de l'Escalonada en fisuras de rocas. Cala d'en Sardina. Puig d'en Corda. Río Sta. Eulalia sobre suelo.

$\because$ Gyroweisia tenuis (Hedw.) Schimp.

Cala d'Aubarca en un talud junto con Trichostomum crispulum y Bryum sp.

Eucladium verticillatum (Brid.) Br. Eur.

Torrent de l'Escalonada sobre rocas. Cala Entrepenyes en a cantilado que rezuma agua. Río Sta. Eulalia cerca del agua.

Trichostomum crispulum Bruch.

Cala d'Aubarca en un talud al pie del acantilado, sobre rocas, en los claros del Rosmarino-Ericion, y bajo Thymus richardii ssp. baueriana en un talud. Torrent de l'Escalonada sobre suelo, sobre rocas y en las fisuras. Cala d'en Sardina sobre suelo. Puig d'en Corda sobre suelo en el Rosmarino-Ericion. Cala.ses Balandres en las físuras del acantilado. Cala Pou des Lleó sobre suelo en la rambla con J. phoenicea v. lycia y $N$. oleander.

Trichostomum brachydontium Bruch.

Cala d'Aubarca bajo R. officinalis y E. multiflora. Torrent de l'Escalonada sobre suelo.

Tortella flavovirens (Bruch.) Broth.

Cala d'Aubarca sobre rocas, en claros del Rosmarino-Ericion sobre suelo, al pie de rocas y bajo $R$. officinalis y E. multiflora. Torrent de l'Escalonada epífito sobre $J$. phoenicea v. lycia y $P$. halepensis, sobre rocas en las fisuras y sobre rocas umbrías. Cala ses Balandres en l as fisuras del acantilado. Cala Pou des Lleó sobre suelo en rambla con J. phoenicea v. lycia y N. oleander. Río Sta. Eulalia sobre piedras. 
* Tortella inflexa (Bruch.) Broth.

Cala Pou des Lleó sobre suelo en rambla con J. phoenicea v. lycia y $N$. oleander.

* Tortella nitida (Lindb.) Broth.

Cala d'Aubarca en talud.

Timmiella barbula (Schwaegr.) Limpr.

Cala d'Aubarca al pie de rocas y sobre suelo debajo de $R$. officinalis y E. multiflo$r a$.

Pleurochaete squarrosa (Brid.) Lindb.

Cala d'Aubarca en los claros del Rosmarino-Ericion.

Grimmia pitardi Corb.

Puig d'en Corda en el Rosmarino-Ericion. Entre Cala Pou des Lleó y Punta d'en Vila sobre suelo debajo de J. phoenicea v. lycia.

* Grimmia pulvinata (Hedw.) Sm.

Torrent de l'Escalonada sobre piedras.

* Funaria hygrometrica Hedw.

Sobre suelo en el Río Sta. Eulalia.

Pohlia camea (Schimp.) Lindb.

Cala Entrepenyes sobre rocas húmedas en el acantilado con Eucladium verticillatum y Leiocolea turbinata.

* Epipterygium tozeri (Grew.) Lindb.

Cala d'Aubarca sobre suelo debajo de Thymus richardii ssp. baueriana.

\section{Bryum gr. erythocarpum}

Puig d'en Corda en los claros del Rosmarino-Erición.

Bryum capillare Hedw.

- Cala d'Aubarca sobre rocas y al pie de rocas húmedas. Torrent de l'Escalonada sobre rocas umbrías.

Scorpiurium circinatum (Brid.) Fleisch. \& Loeske

Cala d'Aubarca sobre rocas umbrías. Torrent de l'Escalonada sobre rocas umbrías. Río Sta. Eulalia sobre piedras.

Homalothecium sericeum (Hedw.) Br. Eur.

Torrent de l'Escalonada sobre suelo, piedras y sobre rocas umbrías.

Brachythecium rutabulum (Hedw.) Br. Eur.

Cala d'Aubarca al pie de rocas y bajo R. officinalis y E. multiflora. Cala d'en Sardina. 
Brachythecium velutinum (Hedw.) Br. Eur.

Torrent de l'Escalonada sobre rocas.

Scleropodium tourettii (Brid.) L. Koch.

Cala d'Aubarca en los claros del Rosmarino-Ericion.

Eurhynchium meridionale (Br. Eur.) De Not. in Picc.

Cala d'Aubarca en talud muy húmedo al pie del acantilado. Torrent de l'Escalonada sobre rocas, sobre suelo y epífito sobre P. balepensis y J. phoenicea v. lycia.

Eurhynchium praelongum (Hedw.) Br. Eur. var. praelongum

Cala d'Aubarca en los claros del Rosmarino-Ericion. Torrent de l'Escalonada sobre piedras.

Eurhynchium praelongum (Hedw.) Br. Eur. var. stokesii (Turn.) Dix.

Cala d'Aubarca en los claros del Rosmarino-Ericion.

Eurhynchium swartzii (Turn.) Curn. in Rabenh. var. swartzii

Cala d'Aubarca en los claros del Rosmarino-Ericion.

Rhynchostegiella curviseta (Brid.) Limpr.

Cala d'Aubarca en los claros del Rosmarino-Ericion. Cala Pou des Lleó sobre suelo en la rambla con I. phoenicea v. lycia y $N$. oleander.

Hypnum cupressiforme Hedw.

Torrent de l'Escalonada sobre suelo y sobre rocas.

\section{FORMENTERA}

\section{HEPATICAS}

Fossombronia sp.

Estéril. Es Pla del Rey sobre suelo arcilloso.

Cephaloziella Baumgartneri Schiffn.

Carretera de la Mol a sobre rocas, sobre tierra y en talud al lado de la carretera.

\section{MUSGOS}

Dicranella varia (Hedw.) Schimp. var. tenuifolia $(=D$. howei)

Es Pla del Rey sobre suelo arcilloso.

Fissidens incurvus Starke \& Röhl

Gruta en el acantilado del Faro de la Mola sobre suelo y rocas. Carretera de la Mola en talud al lado de la carretera. 
* Fissidens exilis Hedw.

Es Pla del Rey sobre suelo arcilloso con Fossombronia sp.

Tortula marginata (Br. Eur.) Spruce

Gruta en el acantilado del Faro de la Mola sobre tierra.

Barbula tophacea (Brid.) Mitt.

Carretera de la Mola sobre suelo, rocas y en talud al lado de la carretera.

Barbula vinealis Brid.

Es Pla del Rey sobre suelo arcilloso.

Gymnostomum calcareum Nees \& Hornsch.

Carretera de la Mola en talud al lado de la carretera.

Eucladium verticillatum (Brid.) Br. Eur.

Gruta en el acantilado del Faro de la Mola. Es Pla del Rey gruta en el acantilado.

Trichostomum crispulum Bruch.

Es Pla del Rey sobre suelo. Carretera de la Mola sobre rocas, suelo y talud al lado de la carretera.

Tortella flavovirens (Bruch.) Broth.

Gruta en el acantilado del Faro de la Mola sobre rocas. Carretera de la Mola sobre suelo y epífito sobre $P$. halepensis.

Tortella inflexa (Bruch.) Broth.

Carretera de la Mola sobre rocas.

Bryum argenteum Hedw.

Es Pla del Rey sobre suelo arcilloso.

Bryum caespiticium Hedw.

Es Pla del Rey sobre suelo arcilloso.

Homalothecium sericeum (Hedw.) Br. Eur.

Carretera de la Mola sobre rocas.

Rhynchostegiella tenella (Dicks.) Limpr.

Carretera de La Mola sobre suelo.

* Rhynchostegiella curviseta (Brid.) Limpr.

Gruta en el acantilado del Faro de la Mola sobre suelo y piedras. 


\section{BIBLIOGRAFIA}

BOLOS, O. de et MOLINIER, R. -1969- Vue d'ensemble de la végétation desiles Baléares. Vegetatio XVII: 251-270. La Haya.

CASARES GIL, A. -1919- Flora Ibérica. Briófitas (1. a parte). Hepáticas. Mus. Nac. Cien. Nat. 1-775. Madrid.

CASARES GIL, A. -1932- Flora Ibérica. Briófitas (2. ${ }^{a}$ parte). Musgos. Mus. Nac. Cien. Nat. 1-434. Madrid.

CASAS, C. -1966- Nueva a portación a la flora briológica balear. Algunos Musgos y hepáticas de las i slas de Ibiza y Formentera. Homenaje en memoria del Profesor J.M. Albareda Herrera. Universidad de Barcelona. Facultad de Farmacia 19-24. Barcelona.

CASAS, C., BRUGUES, M. y CROS, R.M. Contribució al coneixement de l'àrea geogràfica d'alguns briófits. Comunicación en el Simposio de Biogeografia de Sabadell. Abril 1978 (en prensa).

JOVET-AST, S. et BISCHLER, H. -1976- Hépatiques de la Péninsule Ibérique: Enumération, notes écologiques. Rev. Bryol. Lichénol. 42 (4): 931-987.

MAHEU, J. -1912- Exploration et Flore souterraine des cavernes de Catalogne et desiles Baléares. Spelunca 8: 361-465. Paris.

MULLER, P.K. -1954-Die Lebermoose Europas. Leipzig, Yohuson. Reprint Corporation 11365. New York-London.

PERSSON, H. and IMAN, M. -1960- The first find of a Riella in Egypt and some words about the distribution of the genus in the world. Rev. Bryol. Lichénol. 29: 1-9. Paris.

SMITH, A.J.E. -1978- The Moss Flora of Britain and Ireland. Cambridge University Press 1706. Cambridge.

VIVES CODINA, J. -1976- Aproximació a la Flora desl Briofits Balears. 1-53, 10 1. Barcelona. 